\title{
Energy Efficient TRDMA over Multipath Channels
}

\author{
Kavya.T.S \\ M Tech Scholar, ECE Dept \\ SCMS Engineering College \\ Cochin, Kerala, India
}

\author{
Sunil Jacob \\ Professor, ECE Dept \\ SCMS Engineering College \\ Cochin, Kerala, India
}

\begin{abstract}
In recent years, there is an enormous growth in high speed wireless users. Multipath propagation is the transmission of wireless signals from the sender to the receiver through different paths. And these paths are of dissimilar lengths and this result in dissimilar versions of signals reaching at the receiver at dissimilar times. This results in inter-symbol interference by spreading to nearby symbols. Time Reversal Division Multiple Access (TRDMA) is a wireless channel access method which is both energy efficient and low complex is discussed in this paper. TRDMA uses multipath effect to solve the cumbersome issue of inter-symbol interference (ISI). In this technique, energy is concentrated in both spatial and temporal domains. Thus, effective utilization of energy can be made. Multiple user downlink system uses the time reversal structure, where signals from different users are separated by TRDMA. Single transmit antenna with multiple users having single receiving antenna (SISO), Multiple transmit antennas with multiple users having single receiving antenna (MISO) are developed and evaluated. System performance is investigated in terms of achievable sum rate and with outage probability for MISO case. Satisfactory results were obtained through simulation. The simulation for TRDMA was carried out using Matlab software.
\end{abstract}

\section{General Terms}

Wireless communication, Wireless Channel Access method

\section{Keywords}

Time reversal, inter-symbol interference, Time Reversal Division Multiple Access (TRDMA), spatial focussing effect, temporal focussing effect

\section{INTRODUCTION}

Communication is the process of transmission of information from sender to receiver through a guided or unguided medium. Open space is used as signal propagating medium for wireless communication. Recently, there is a steady increase in number of wireless users which necessitates the need for future broadband communications. Inter-symbol interference (ISI) due to multipath effect makes high speed communication a very difficult task. Different techniques were used to resolve ISI, such as channel equalization, diversity, coding, multicarrier modulation techniques (such as OFDM), although the performance is well acceptable, it results in high complexity for end user equipments [1]-[4].

On the other hand, time-reversal (TR) signal transmission technique can provide low-complexity energy-efficient communications. Using the nature of multi-path environments they can concentrate energy in both the spatial and temporal domains providing energy-efficient communications. With time reversal methods, the multipath channel can be used as a merit. A pilot signal can be transmitted by the receiver to the base station. The base station records that signal at an antenna array. Those signals are time reversed and are saved as signature signals. When a data is to be transmitted by the base station to the receiver (user), the signature signals can be used. The new signal will travel back through the same channel as it was received. The multipath effects that affected the signal in the forward path will have an inverse effect in the reverse path. As a result, there is destructive interference in almost all locations while the receiver will have a strong signal due to constructive interference.

\section{TIME REVERSAL THEORY}

Focusing on a reflective target in an inhomogeneous media is somewhat difficult. Time reversal mirrors (TRM) were used to obtain focussing. TRM is an array of transmit- receive transducers. TRM transmits a signal to the target and the signals are reflected by the target. TRM receives and stores the reflected signals. And they are reversed in time and are reemitted.

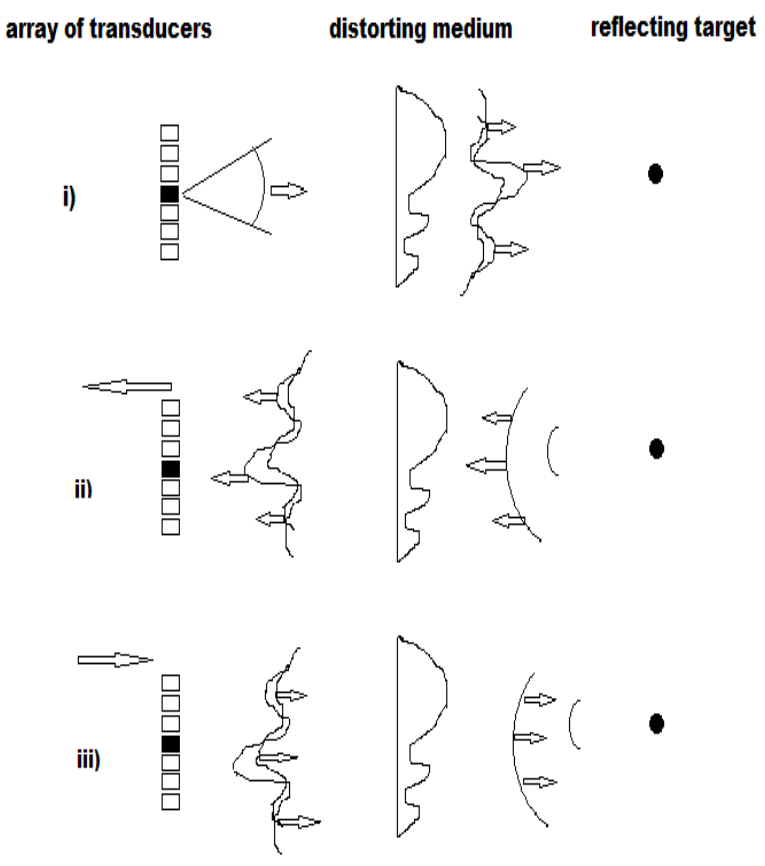

Figure 1: Focusing using TRM method

This procedure allows converting a divergent signal to a convergent signal focussing on the source. TRM method provides the ability to focus through an inhomogeneous medium on a reflective target whose position is unknown. The process can be iterated in order to make a selective focussing in case of many targets [5]-[8]. 
Focussing using TRM can be done in three steps. In the initial step, array of transducers sends the signal to the reflective target. Echoes reflected by the target are distorted by the medium and stored in the array. In the final step, the signal is time reversed and is retransmitted through the medium and focusses on the target. This is illustrated in figure1. The time reversal theory was simulated (figures 2 and 3 ) and was found that the parallel procedure using TRM helps to focus the signal on the target.

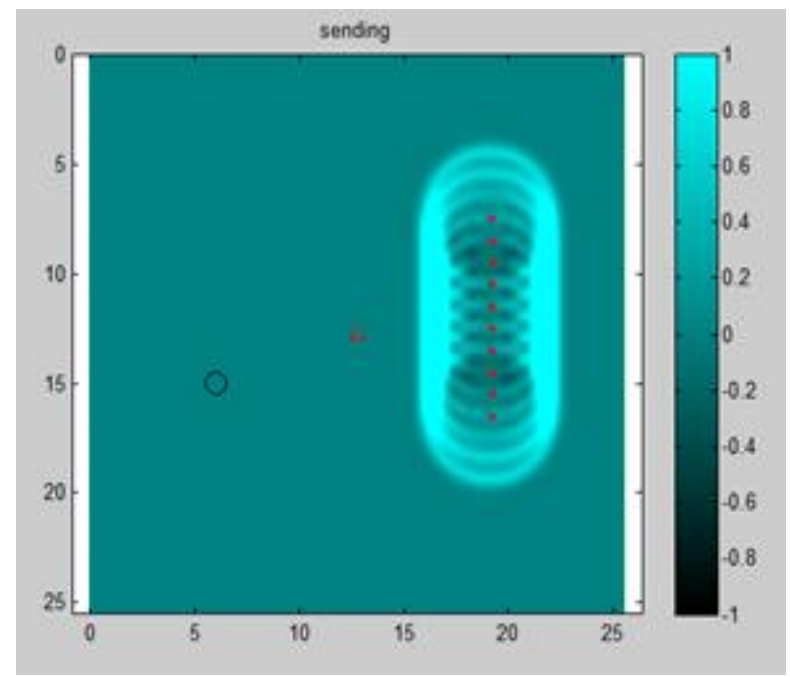

Figure 2: Initial step of TRM focussing

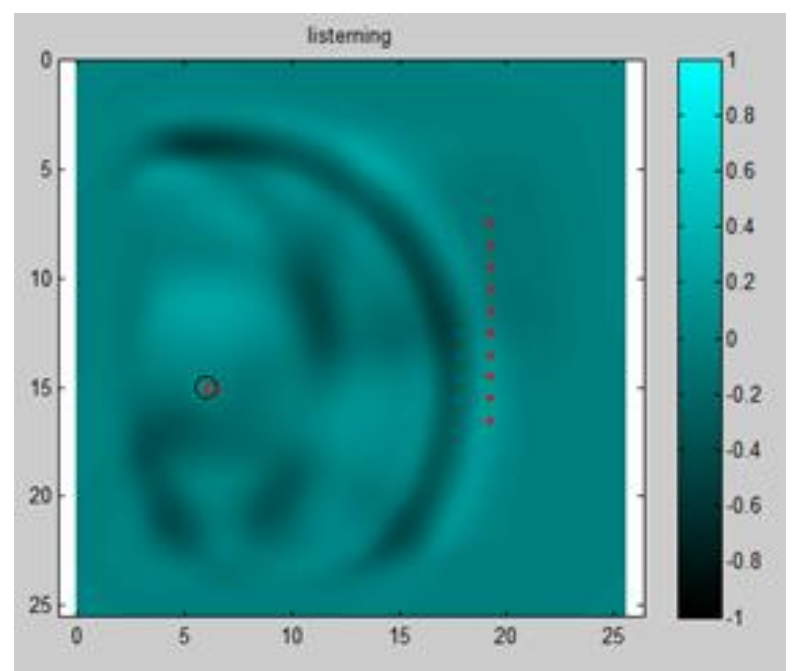

Figure 3: Final step of TRM focusing

Mathematically, Let $y(t)$ be the signal transmitted by the target to the TRM and $h(t)$ be the impulse response of the channel. Then, the received signal by the $i$ th transducer in the TRM array,

$$
r_{i}(t)=y(t) \otimes h(t)
$$

In frequency domain,

$$
\mathrm{R}_{\mathrm{i}}(\mathrm{f})=\mathrm{Y}(\mathrm{f}) \mathrm{H}_{\mathrm{i}}(\mathrm{f})
$$

Time reversing gives $r_{i}(-t)$ and in frequency domain $R_{i}^{*}(f)$. The channel has same response $h(t)$ in reverse direction, transmitting $\mathrm{r}(-\mathrm{t})$ back through the channel gives,

$$
\mathrm{Z}(\mathrm{f})=\mathrm{R}_{\mathrm{i}}{ }^{*}(\mathrm{f}) \mathrm{H}_{\mathrm{i}}(\mathrm{f})
$$

$$
\begin{aligned}
& =Y^{*}(f) H_{i}^{*}(f) H_{i}(f) \\
Z(f) & =A Y^{*}(f)
\end{aligned}
$$

Where, $\mathrm{A}$ is a scaling factor which symbolizes the gain of filter.

\section{TIME REVERSAL DIVISION MULTIPLE ACCESS (TRDMA)}

Time reversal mirrors (TRM) are used for achieving focusing of multipath signals on a target. Time Reversal Division Multiple Access (TRDMA) uses TRM so as to provide low complexity and energy efficient communication. This method makes full use of nature of multipath environments which is a boon for other techniques.

Time reversal method is based on a characteristics of wave equation, which is known as reciprocity.ie, given a solution for the wave equation, then the time reversal (taking negative time, if the solution is complex then complex conjugate is taken)of that solution is also a solution for the wave equation.

\subsection{Fundamental TR Communication System}

TR communication fundamentally consists of two phases: Recording phase (Channel probing phase) and Transmission phase. Two assumptions are considered for TR communication; they are channel reciprocity and channel stationarity.

Channel reciprocity-impulse response of forward channel and backward channel are assumed to be same.

Channel stationarity: impulse response of a channel is assumed to be a constant for atleast one recording and transmission cycle.

Consider a single user TR communication. When transceiver A wants to send data to transceiver $\mathrm{B}$, in TR communication first transceiver $B$ sends a pilot signal of length $\mathrm{Tp}$ to transceiver A through multipath channel. Transceiver A records the received signal which is a convolution of pilot signal and impulse response of the channel. And then transceiver A time reverses the received signal and saves it as a signature for transmitting data from A to B. This is known recording phase.

At transceiver $\mathrm{A}$, there are $\mathrm{X}[\mathrm{k}]$ symbols which are to be transmitted to $\mathrm{B}$. The rate back off factor (D) is used for making the symbol rate equal to the chip rate. Then, the time reversed signature is sinked with $\mathrm{X}[\mathrm{k}]$, and is transmitted through the same channel to B. Transceiver B desamples the received signal to detect the symbols $\mathrm{X}[\mathrm{k}]$.This is called transmission phase. These two phases are illustrated in figure4.

By using channel reciprocity, the reemitted TR waves can retrace the path causing a spiky signal power only for the intended user. This is called spatial focusing effect. TR influences the multipath channel as a matched filter and focuses the wave in time domain. This is known as temporal focusing effect [11]. And this is how TRDMA becomes energy efficient communication system that eliminates ISI. Any features of the channel are not needed to be known for TR signal processing method. One only needs to know whether the medium is reciprocal or not. 
In transmission phase, the inputs are first up sampled by rate back off factor (D), they are fed to TRM . The output of TRMs

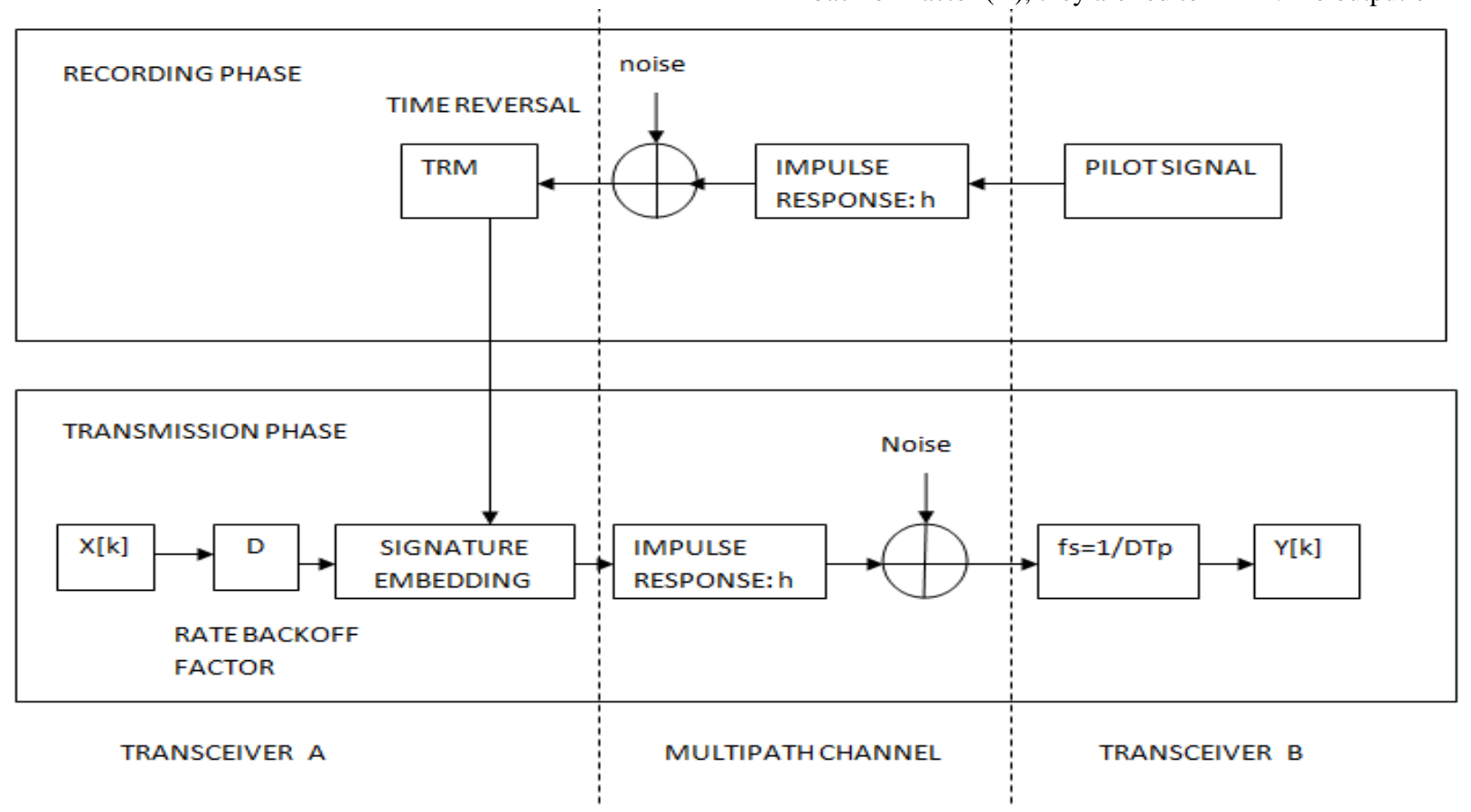

Figure 4: TR Communication System

\subsection{SISO TRDMA}

Single Input Single Output (SISO) TRDMA system contains base station with single transmit antenna and multiple users with single receiving antenna. During channel probing phase of SISO TRDMA, $\mathrm{N}$ users take turns to transmit a pilot signal to base station.TRM at base station records the channel response of each link and stores the time reversed version of each response for transmission phase [13]. are the combination of up sampled data with TRM waveform.

All the outputs from TRMs are summed up and transmitted into mobile channel. Embedding with time reversed waveform helps the waveform to reach the meant user. Single tap gain adjustment is done at the receiver to the received signal to recover the signal and down samples it with a factor $\mathrm{D}$. This is illustrated in figure 5 .

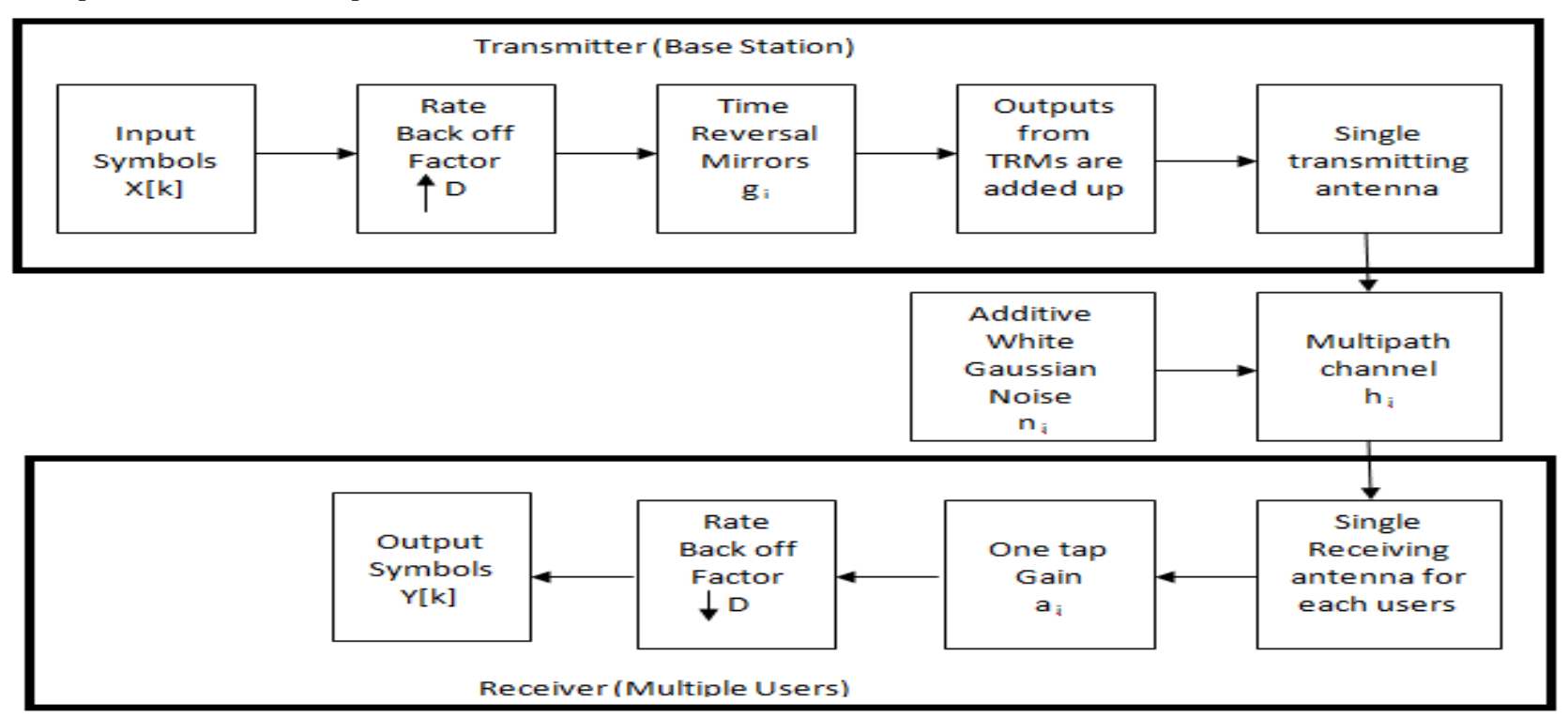

Figure 5: SISO TRDMA Transmission Phase 


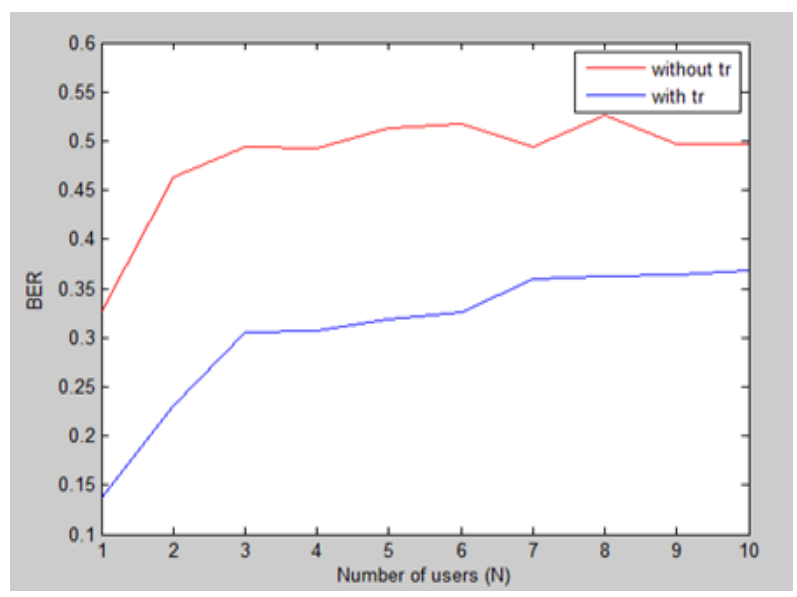

For SISO TRDMA bit error rate was found to be less for data with time reversal than data without time reversal.

\subsection{MISO TRDMA}

Multiple Input Single Output (MISO) TRDMA system contains base station with multiple transmitting antenna and multiple users with single receiving antenna. In case of MISO, each antenna at the transmitting base station is similar to the single antenna base station in the SISO case [13].

Figure 6: Bit error rate for SISO TRDMA

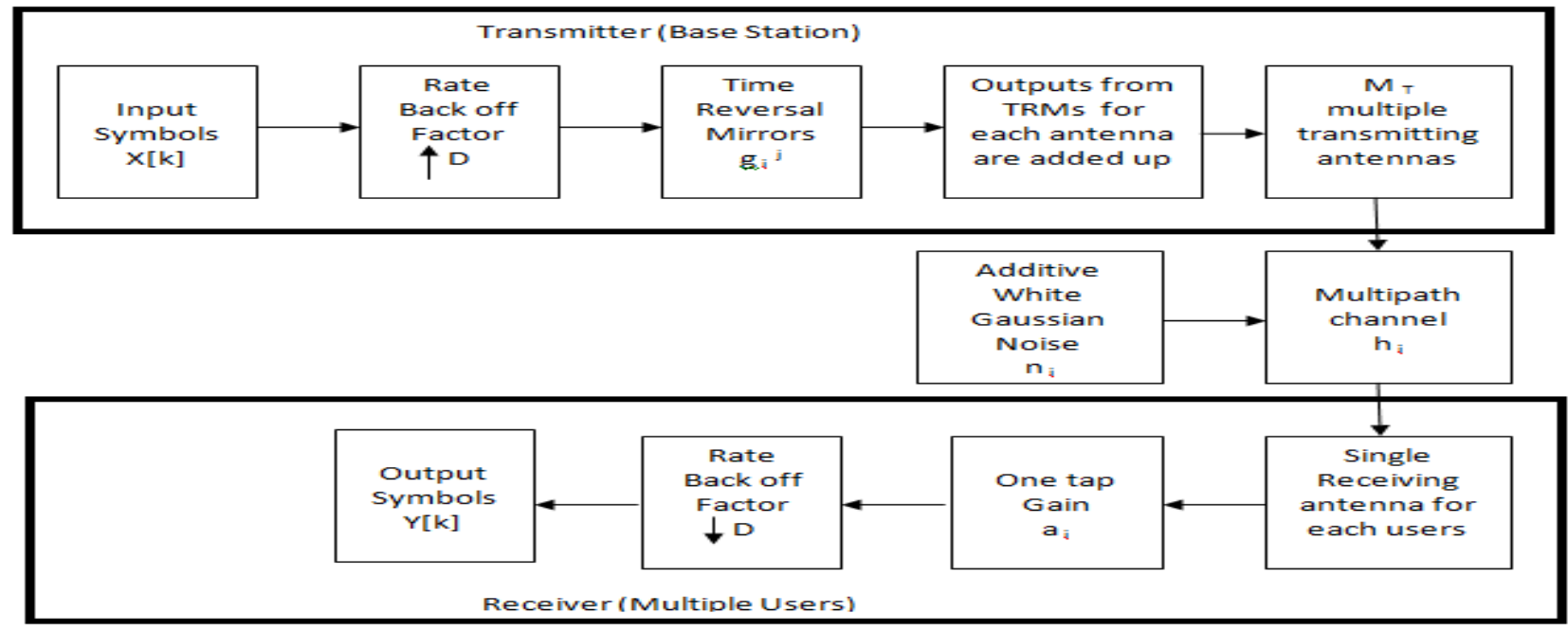

Figure 7: MISO TRDMA Transmission Phase

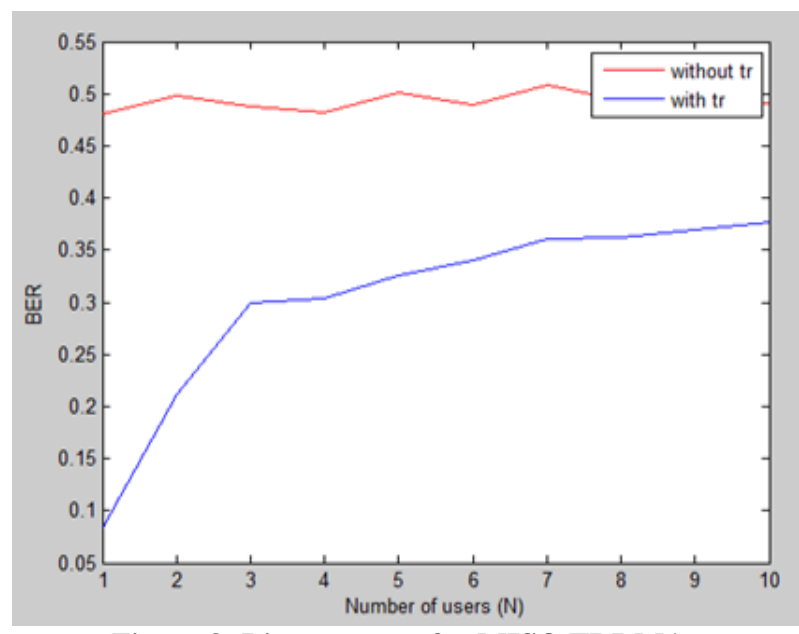

Figure 8: Bit error rate for MISO TRDMA

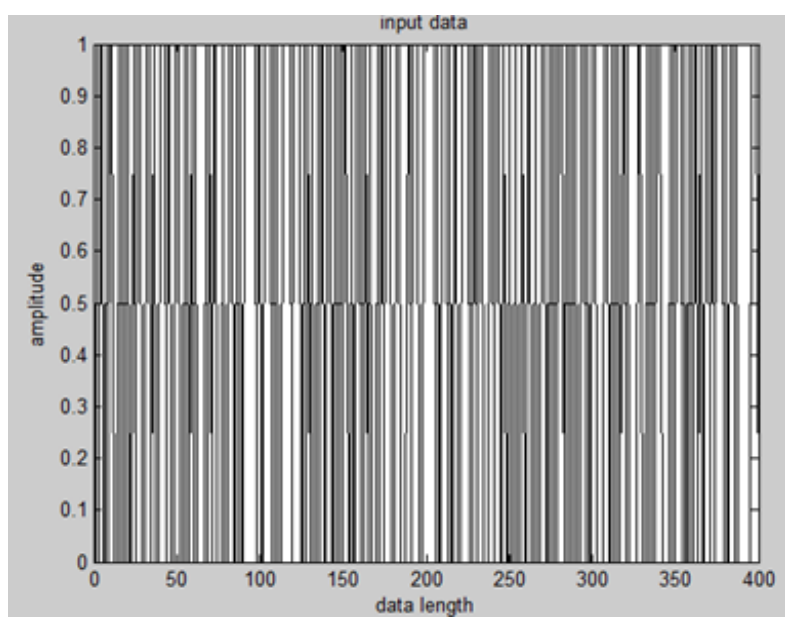

Figure 9(a) Data that is transmitted to the user

Similar to SISO, for MISO also bit error rate for data with time reversal is less than that of data without time reversal.

Figure 9 reveals the exact difference between the signal received by a user in a MISO case using with and without time reversal method. 


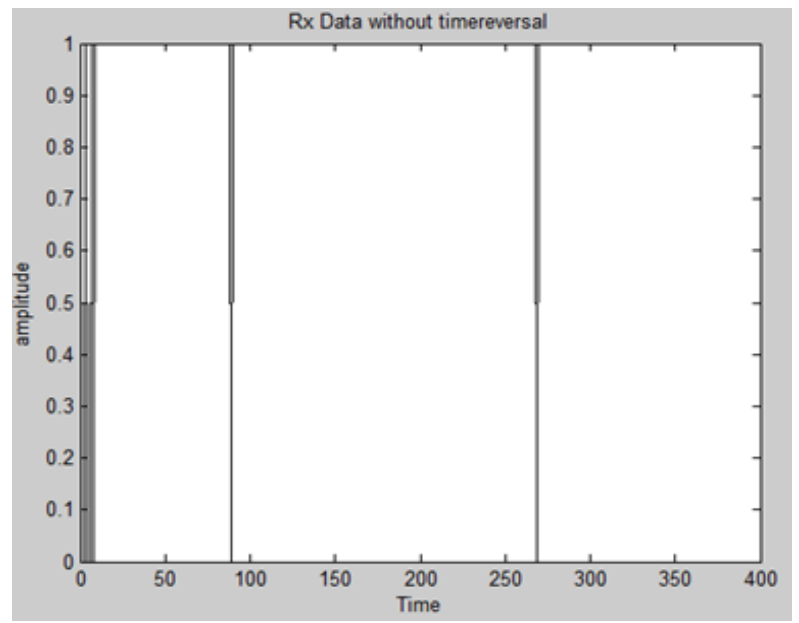

Figure 9(b) Data received by the user without using TR

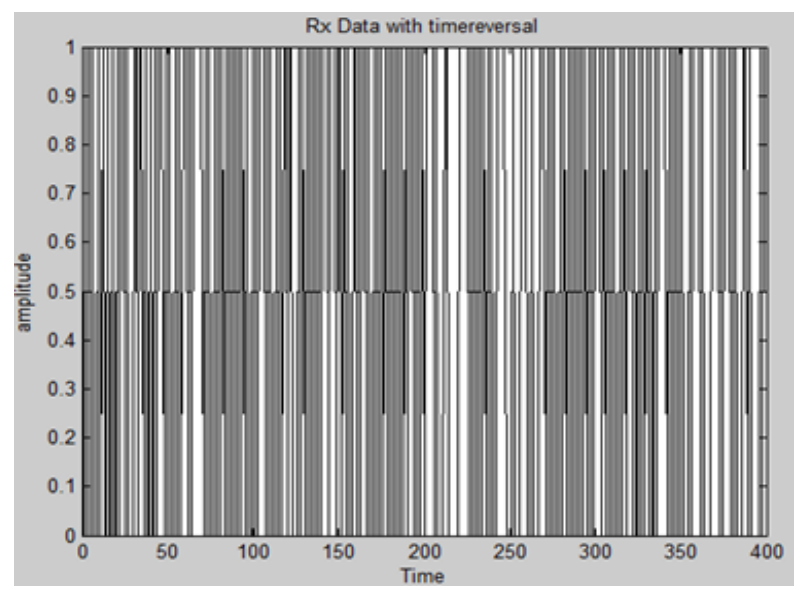

Figure 9(c) Data received by the user using TR

Figure 9: Data transmitted and received using with and without time reversal

Figure 9 depicts that the data received by the user using time reversal method is almost similar to that was transmitted to the user.

The achievable sum rate or transmission rate is defined as the mutual information between the transmitted and the received signals. The achievable sum rate for $\mathrm{N}$ users where $\mathrm{D}$ is the rate back off factor is given by ,

$$
\mathrm{R}=\frac{\eta}{\mathrm{D}} \sum_{\mathrm{i}=1}^{\mathrm{N}} \log (1+\operatorname{SINR}(\mathrm{i}))
$$

Where, $\eta$ is a factor that describes the portion of the transmission phase. Signal to interference plus noise ratio (SINR) can be defined as the ratio of the average signal power to the average interference plus noise power.

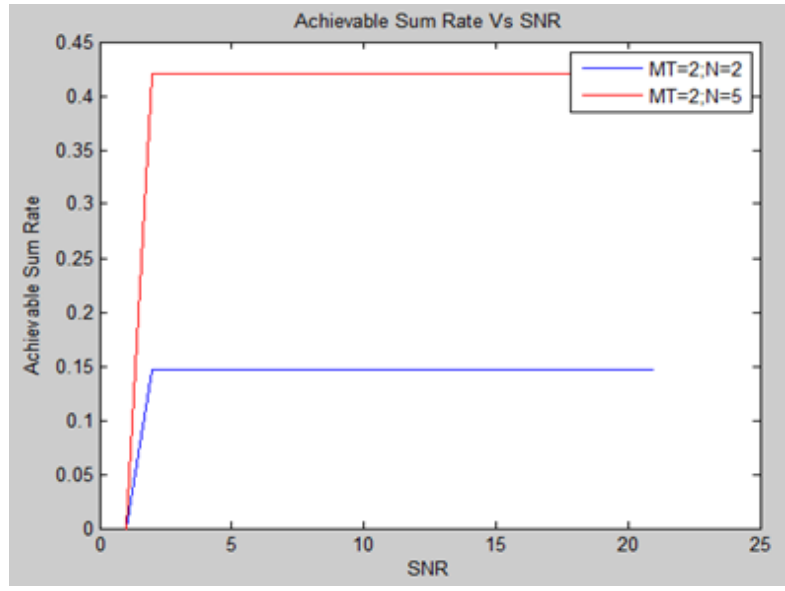

Figure 10: Achievable sum rate for MISO case

Keeping number of transmitting antennas as a constant and by varying the number of users, it was found that (from figure 10), as the number of users increases, achievable sum rate also increases.

Outage probability is the probability that an outage will occur within a specified time period, where outage is a condition in which user is deprived of service by the system. Outage probability can be found by,

$\mathrm{P}_{\text {out }}=\operatorname{Pr}\left\{\frac{1}{\mathrm{~N} * \mathrm{D}} \sum_{\mathrm{i}=1}^{\mathrm{N}} \log (1+\mathrm{SINR}(\mathrm{i}))<R\right\}$

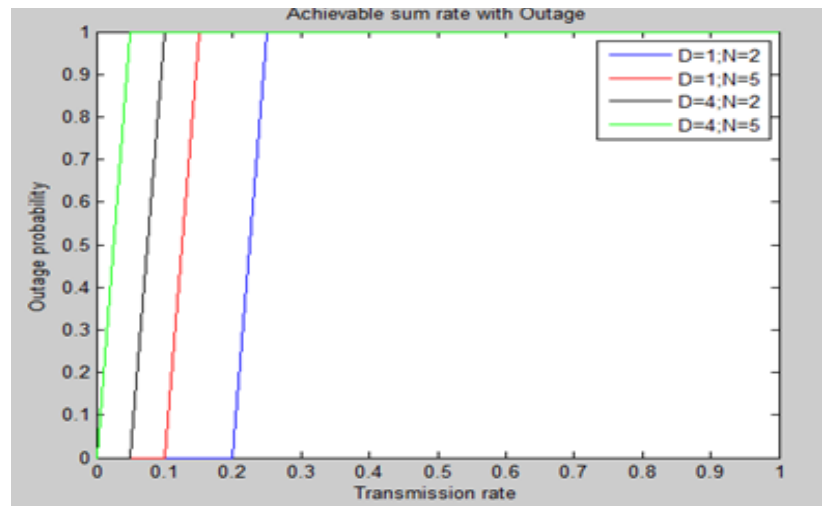

Figure 11: Achievable sum rate with outage for MISO case

From figure 11, it can be inferred that a larger D results in reduced individual achievable rate of the user and a larger $\mathrm{N}$ also reduces the individual achievable rate of user with same outage probability.

\section{MERITS OF TRDMA}

- Both downlink and uplink can support transmission of multiple users.

- Efficiently alleviate inter-symbol interference (ISI)

- TRDMA is an energy efficient communication system, since it can concentrate energy in both spatial and temporal domains.

- Using location specific signature, security can be provided in TRDMA [12].

- Low complex 


\section{COMPARISON WITH OFDM}

Orthogonal Frequency Division Multiplexing (OFDM) has high spectral efficiency, since it can provide high data rate even with limited bandwidth. High computational complexity, high energy consumption, high peak to average power ratio [10] and interference at transmitter and receiver are other features of OFDM. When bandwidth is wide enough, advantage of high spectral efficiency becomes less significant and high complexity, high energy consumption at both transmitter and receiver becomes major drawbacks. Hence, OFDM is not a good solution when bandwidth is larger than $4 \mathrm{G}$ systems.

For comparing the complexity, exclude the common components in both OFDM and TRDMA such as automatic gain control (AGC), power allocation etc. The distinctive components used in OFDM are IFFT at the transmitter and FFT at the receiver. When number of subcarriers $\mathrm{N}$ is a power of 2, according to radix 2 Cooley Tukey algorithm, FFT requires $\mathrm{N} / 2 * \log _{2} \mathrm{~N}$ complex multiplications and $\mathrm{N}^{*} \log _{2} \mathrm{~N}$ complex additions. When there are $\mathrm{M}$ receivers, an $\mathrm{N}$ point IFFT is required at the transmitter and $\mathrm{M} ; \mathrm{N}$ point FFT is required at the receiver.

Similarly, waveform design and signal mixing are the distinctive components in TRDMA. Waveform design is needed to be computed only when the channel alters, not at all slots. Therefore, complexity comes from signal mixing at the transmitter. Signature waveform gets added with the symbols at the transmitter in signal mixing. Hence there are no complex multiplications; only complex additions are present at the transmitter side. Since single tap detection is done at the receiver, there is no complexity at the receiver side. From, this it is clear that TRDMA is computationally less complex than OFDM systems.

\section{CONCLUSION}

Time Reversal Division Multiple Access for wireless communication in multipath environment was discussed in this paper. Spatial and temporal focussing effect makes TRDMA energy efficient. Focussing ability of TR method was simulated and positive results were obtained. Simulation results confirm that BER for SISO and MISO case for data with time reversal is less than that without time reversal. Performance evaluation for MISO was carried out in terms of achievable sum rate and outage probability. Simulation results showed that probability of a system that moves to outage occurs quickly with increase in number of users and rate back off factor. Computational complexity of TRDMA compared with OFDM was very low. TRDMA provides low complexity energy efficient communication which makes full use of nature of multipath environments. This method doesn't require any complex signal processing and equalization.

With the inherent nature to fully harvest energy from the surrounding environment by exploiting the multi-path propagation, the TR system has a potential of reduction in power consumption and interference alleviation, which means that TR system can provide better battery life and support multiple concurrent active users. By adjusting the waveform and rate back off factor, various QoS options can be easily supported in TR systems. Finally, the unique location-specific signature in TR system can provide additional physical-layer security and thus can enhance the privacy and security of customers. All these merits shows that TR technique is an ideal paradigm for IoT (internet of things). Based on the discussions, it can be clearly stated that TRDMA can be a promising technology for future broadband communication such as $5 \mathrm{G}$.

\section{ACKNOWLEDGEMENT}

I would like to acknowledge the inspiration given by Dr. Sunil Jacob, who guided me throughout the project and the motivation given by Mrs. Saira Joseph in completing this paper on time.

\section{REFERENCES}

[1] Theodore.S.Rappaport, $2^{\text {nd }}$ edition Pearson,2001,Wireless communication- Principles and Practice

[2] J. G. Proakis, 4th edition, McGraw-Hill, 2001 , Digital Communications

[3] G. L. Stuber, Principles of Mobile Communications, 2nd edition., Kluwer, 2001

[4] D. Tse and P. Viswanath, Fundamental of Wireless Communication, Cambridge University Press, 2005.

[5] M. Fink, C. Prada, F. Wu, and D. Cassereau,1989, Self focusing in inhomogeneous media with time reversal acoustic mirrors, IEEE Ultrasonics Symp., vol. 1, pp. 681-686

[6] C. Prada, F. Wu, and M. Fink, 1991,The iterative time reversal mirror: a solution to self-focusing in the pulse echo mode, J. Acoustic Society America, vol. 90, pp. 1119-1129, 1991.

[7] C. Dorme and M.Fink,1995,Focusing in transmit-receive mode through inhomogeneous media: the time reversal matched filter approach, J. Acoustic Society America, vol. 98, no. 2, part 1, pp. 1155-1162

[8] A. Derode, A. Tourin, J. de Rosny, M. Tanter, S. Yon, and M. Fink, 2003, Taking advantage of multiple scattering to communicate with time reversal antennas,Phys. Rev. Lett., vol. 90, pp. 014301$1-014301-4$

[9] Persefoni Kyritsi, George Papanicolaou, Patrick Eggers, Alex Oprea, 2004, Time Reversal Techniques for Wireless Communications, IEEE.

[10] Jungwon Lee, Hui-Ling Lou, Dimitris Toumpakaris, John M. Cioffi, December 2006 , SNR analysis of OFDM systems in the presence of Carrier frequency offset for fading channels, IEEE Transactions on wireless communications, vol. 5, No. 12 .

[11] H. T. Nguyen, I. Z. Kovacs, and P. C. F. Eggers, Nov. 2006,A time reversal transmission approach for multiuser UWB communications, IEEE Trans. Antennas Propagation., vol. 54, no. 11, pp. 3216-3224,

[12] Beibei Wang, Yongle Wu, Feng Han, Yu-Han Yang, and K. J. Ray Liu, September 2011,Green Wireless Communications: A Time-Reversal Paradigm, IEEE Journals on selected areas in communications,vol.29, no.8.

[13] Feng Han, Yu-Han Yang, Beibei Wang, Yongle Wu, K. J. Ray Liu, July 2012 ,Time-Reversal Division Multiple Access over Multi-Path Channels, IEEE Transactions on communications, vol.60, No.7. 